\title{
Flapping Wing Aerodynamics of a Numerical Biological Flyer Model in Hovering Flight
}

\author{
Hikaru Aono ${ }^{\mathrm{a}, *}$, Hao Liu ${ }^{\mathrm{b}}$ \\ ${ }^{a}$ Institute of Space and Astronautical Science/Japan Aerospace Exploration Agency, 3-1-1 Yoshinodai, \\ Sagamihara 252-5210, Japan \\ ${ }^{b}$ Chiba University, 1-33 Yayoi-cho, Chiba 263-8522, Japan
}

\begin{abstract}
Flapping flyers showcase excellent flight performances under many flight environments. In particular, hovering is a miracle of insects that can be seen for most of sizes of flying insects. Understanding of sizing or Reynolds numbers effects in hovering flights on the aerodynamics is not only of interest to the micro-air-vehicle ommunity but also of importance to comparative morphologists. In this study, a computational study of such size effects on insect hovering aerodynamics is conducted, which is performed using an integrated numerical framework consisting of the modeling of realistic wing-body morphology, the modeling of flapping-wing and body kinematics, and an in-house NavierStokes solver. Computational results of four typical insects in hovering flight including a thrips, a fruitfly, a honeybee, and a hawkmoth over a wide range of Reynolds numbers from $O\left(10^{1}\right)$ to $O\left(10^{4}\right)$ are presented. Furthermore the correlation among the near-and far-field flow features, the aerodynamic force production, and the wing kinematics is highlighted.
\end{abstract}

Keywords: Flapping wing, Unsteady aerodynamics, Leading edge vortex, insect hovering flight

\footnotetext{
*Corresponding author. Tel.: +81-050-3362-3751

Email address: aono@flab.isas.jaxa.jp (Hikaru Aono)
} 


\section{Introduction}

Birds, bat, and insect flight has fascinated humans for many centuries [1]. In Earth nearly a million species of flying insects, and of the living 13,000 birds and mammals, and 1,000 bats have taken to the skies. With respect to maneuvering a body efficiently through space, birds represent one of nature's finest locomotion experiments. While aeronautical technology has advanced rapidly over the past centuries, nature's flying machines, which have evolved over 150 million years, are still impressive. Considering that humans move at top speeds of 3-4 body lengths per second, a supersonic aircraft such as the SR-71 traveling near Mach 3 covers about 32 body lengths per second, it is remarkable that a common pigeon frequently attains 75 body lengths per second, and various species of swift are even more impressive, over 140 body lengths per second. The primary reasons for such superior maneuvering and flight characteristics include scaling laws with respect to a vehicle's size, as well as intuitive but highly developed sensing, navigation, and control capabilities. As McMasters and Henderson put it, humans fly commercially or recreationally, but animals fly professionally [2].

Compared to flapping wings, conventional airplanes with fixed-wings are relatively simple; the forward motion relative to the air causes the wings to produce lift. However, in biological flapping flight the wings not only move forward relative to the air; they also flap up and down, plunge, and sweep [1]. While, in earlier days of flight of efforts regarding the flapping wing aerodynamics, much of the analysis is based on the analogy to fixedwing counterpart, it was known that this approach encounters qualitative difficulties, for example, the airplane were considered as a similar size of a bee, moving as slowly as a bee, could not fly. However, bees can fly. This story suggested in simple fashion the implied conclusion-that the theory of fixed wing aerodynamics cannot explain certain critical aspects of the flapping wing aerodynamics. The aforementioned framework essentially considers the flapping wing dynamics as a series of snapshots by neglecting the influence of the aerodynamics and wing motion at an earlier moment on the aerodynamics at a later time, based on the so-called quasi-steady approach [3]. In reality, in order to generate the desirable lift and thrust under various conditions, a small flyer can often benefit from manipulating unsteady fluid flows via flapping wing.

Commonly, flapping wing aerodynamics characterizes time-dependent wing motions, 
flexible wing structure, and low Reynolds numbers (characterizing the relative importance between inertia and viscous effects of fluid). As highlighted in Fig 1, it is observed that the Reynolds numbers are the order of $10^{1}$ for a tiny thrips up to order of $10^{4}$ for a moth. Many studies have significantly contributed to understanding aerodynamic mechanisms of the flapping flight. For instance, numerical and physical experiments with dynamically-scaled biological rigid wing models under hovering condition reveal several unsteady aerodynamic mechanisms, namely, delayed stall of leading-edge vortex (LEV) [4], active wing rotation [5], an interaction between the LEV and tip vortex (TV) [7], as well as recapturing vortices in a wake $[5,6]$. Most of them substantially play a role in enhancing lift generation in the flapping flight while some of them such as the wake capture and tip vortices may lead to a decrease in the aerodynamic performance when the wing orientation and the vortical structure are not well coordinated. Moreover, the effects of wing flexibility on unsteady aerodynamic mechanisms are an ongoing topic and recently actively investigated [8]. More importantly, the effectiveness of these unsteady mechanisms is strongly linked with resultant flapping wing movement including passive wing motion, the Reynolds number, and the flight environment. Therefore, a concrete explanation and implication on the unsteady aerodynamic mechanisms still remains unclear, especially in terms of the effects of sizing or $R e$ and the wing flexibility. Furthermore modeling of morphology and kinematics based on real insects and birds is no doubt a must but have not been studied systematically yet, which is not only of great interest to the micro air vehicle (MAV) community but also of importance to comparative morphologists when considering how physics constrains biological design $[1,8]$.

The objective of present paper is twofold: (i) to provide an insight of how different morphology and kinematics in insect flight are; (ii) to show how morphology and kinematics influence the aerodynamics in terms of either sizing or Reynolds number.

\section{Material and methods}

\subsection{Numerical framework of flapping flight: a biology-inspired dynamic flight simulator}

Current study utilizes a numerical framework for analyzing flapping wing flight, namely, a biology-inspired dynamic flight simulator. This simulator is versatile and established based on the modeling of realistic wing-body morphology, realistic flapping-wing 
and body kinematics, and unsteady aerodynamics in flapping flights. A morphological model is built based on an effective differential geometric method for reconstructing geometry of and an in-house grid generator for the wing and body; and a multi-blocked, overset-grid method is utilized to deal with complicated wing-body geometries and timedependent flapping movements with multiple degrees of freedoms. A kinematic model is constructed to be capable of mimicking the realistic wing-body kinematics of flapping flight; and an efficient analytical method combined with three coordinate systems is employed for the dynamic re-gridding. A fortified finite-volume method-based NavierStokes solver for the dynamically moving multi-blocked, overset-grid system is developed and verified to be self-consistent by a variety of benchmark tests. The evaluation of flapping energetics is established on both instantaneous and period-averaged inertial and aerodynamic forces, torques, and powers.

Furthermore this simulator has been validated by the comparisons of aerodynamic force-production with experimental measurements in terms of the instantaneous and flapping cycle averaged lift and drag forces. The results of four typical insect hovering flights (a hawkmoth, a honeybee, a fruitfly, and a thrips) over a wide range of Reynolds numbers from $O\left(10^{1}\right)$ to $O\left(10^{4}\right)$ have been demonstrated its feasibility in accurately modeling and quantitatively evaluating the unsteady aerodynamic mechanisms in insect flapping flight. Further details on description of numerical methods can be found in Aono et al. [7], Liu and Aono [9], Liu et al. [10], Liu and Kawachi [11], and Liu [12].

\subsection{Modeling of morphology and kinematics of a biological flyer}

Wing-body morphological models of four typical insects, namely a hawkmoth, a honeybee, a fruitfly, and a thrips, are constructed. In the morphological modeling the special attention is received for the fact that multiple morphologies of a two- or four-winged body and unique wing-body geometry feature in biological flapping flights. To deal with such complexity a chimera grid scheme-based overset-grid method is adopted. The grid is clustered to the wing-and body-surface with the minimum grid spacing adjacent to the wing surface controlled by a formula $0.1 c_{m} / \sqrt{R e}$, where $c_{m}$ is mean chord length and $R e$ is the chord-based Reynolds number. The body grid is sufficiently large, which has a distance between the body surface and the outside boundary of approximately twenty 
times the mean chord length $c_{m}$, whereas the wing grid has an outside boundary of two times the mean chord length $c_{m}$.

Fig 2 illustrates computational grid systems of four typical insects. Kinematics of flapping flight, in general, consists of wing beat and body kinematics. The insect body, if it is assumed to be rigid during flapping motion, can be represented by the inclination of the body to the ground (or, body angle $\chi$ ) and the stroke plane angle $(\beta)$, which varies according to the variation in flight speeds. While the wing-beat kinematics can be described by three basic rotational angles within the stroke plane as shown in Fig 3. Here, a general definition of the positional angle (or the stroke angle), the elevation angle (or the deviation angle) and the feathering angle in terms of the geometric angle of attack of a wing, all in degrees, are expressed using the Fourier series, such as:

$$
\begin{aligned}
& \varphi(t)=\sum_{n=0}^{3} \varphi_{c n} \cos (n \omega t)+\varphi_{s n} \sin (n \omega t) \\
& \vartheta(t)=\sum_{n=0}^{3} \vartheta_{c n} \cos (n \omega t)+\vartheta_{s n} \sin (n \omega t) \\
& \alpha(t)=\sum_{n=0}^{3} \alpha_{c n} \cos (n \omega t)+\alpha_{s n} \sin (n \omega t)
\end{aligned}
$$

where $t$ is a dimensional time, $\omega$ the angular frequency, $n$ an integer varying from 0 to 3 , and the coefficients $\varphi_{c n}, \varphi_{s n}, \vartheta_{c n}, \vartheta_{s n}, \alpha_{c n}, \alpha_{s n}$ can be determined from the measured kinematic data [13] [14]. Fig 4 depicts hovering kinematic models of four insects.

Consider flying in the air $\rho_{\text {air }}$, the mean chord length $c_{m}$ as the reference length $L_{r e f}$, the mean wing tip velocity $U_{t i p}$ in hovering flight the reference velocity $U_{r e f}$, which is proportional to $U_{t i p}=2 \Phi f R$, where $\Phi$ is the wing tip peak-to-peak amplitude, $f$ is the flapping frequency, and $R$ is the wing length. Therefore, the Reynolds number $(R e)$ in hovering flight can be defined and reformed as

$$
R e=\frac{\rho_{r e f} U_{r e f} L_{r e f}}{\mu_{r e f}}=\frac{\rho_{a i r} 2 \Phi R f c_{m}}{\mu_{a i r}}=\frac{4 \Phi f R^{2}}{\nu_{a i r} A R}
$$

where the aspect ratio $A R$ is in a form of $A R=(2 R)^{2} / 2 S$, with total wing area of $S=2 R c_{m}$, and $\nu_{\text {air }}$ the kinematic viscosity of air $\left(1.5 \times 10^{-5} \mathrm{~m}^{2} / \mathrm{s}\right)$. Variation in the flapping amplitude $(\Phi)$ and the aspect ratio $(A R)$ due to the change of the insect size is not significant 
in comparison with the variation in the flapping frequency $(f)$ and the wing length $(R)$ [15]. This characteristic of morphology and wing kinematic of biological flyer results in a wide $R e$ range from $O\left(10^{1}\right)$ for tiny insects, $O\left(10^{2}\right)$ for fruitflies, $O\left(10^{3}\right)$ for honeybees, butterfies, beetles, and dragonflies, and up to $O\left(10^{4}\right)$ for hawkmoths and large insects. The reduced frequency $(k)$ is another important non-dimensional parameter that normally characterizes a ratio of timescales between the flapping motion and the reference velocity, is defined in case of hovering flights, such as:

$$
k=\frac{\omega L_{r e f}}{2 U_{r e f}}=\frac{\pi}{\Phi A R}
$$

Hawkmoth hovering: $R e=4,000-8,000$

A realistic wing-body morphological model is built based on a hawkmoth, Agrius convolvuli [9]: a body length is $5.0 \mathrm{~cm}$, a wing length $(R)$ is $4.83 \mathrm{~cm}$, and a mean chord length $\left(c_{m}\right)$ is $1.83 \mathrm{~cm}$, respectively. A uniform wing thickness $\left(0.012 c_{m}\right)$ is assumed for the two wings, which resembles the real hawkmoth-wing morphology; and a virtual distance of the wing length (approximately $0.03 \mathrm{~cm}$ ) is added to the wing-base to avoid the attachment of the wing on the body surface, which could improve the numerical convergence but insignificantly affect the results in the hovering flight. Kinematic models are based on the experimental data of a hovering hawkmoth, Manduca Sexta [13]: positional angle (stroke angle), feathering angle (deviation angle) and elevation angle (geometric angle of attack) are plotted as in Fig 4 (a). Grid system of the wing-body hawkmoth model as illustrated in Fig 2 (b-1) is composed of three-blocked overset grids (right wing grid: 62,775 points, left wing grid: 62,775 points, body grid: 200,925 points). O-O type structured body-fitted grid is employed. The body angle $\chi$ is 39.8 deg., the stroke plane angle $\beta$ is 15 deg., the wing stroke amplitude $\Phi$ is 114.6 deg., and the flapping frequency $f$ is $26.1 \mathrm{~Hz}$. Therefore, the Reynolds number $(R e)$ and reduced frequency $(k)$ is calculated to be approximately 6,300 and 0.298 .

Honeybee hovering: $R e=1,000-2,000$

A realistic wing-body model of a honeybee, Apis mellifera [9], is constructed as illus- 
trated in Fig 2 (b-2): a body length of $10.0 \mathrm{~mm}$, a wing length $(R)$ is $9.7 \mathrm{~mm}$, and a mean chord length $\left(c_{m}\right)$ of $2.39 \mathrm{~mm}$. A $0.012 c_{m}$ flat plate wing model is employed. Total number of grid points of each wing and body are 62,775 and 200,925. Kinematic models are based on the experimental data of a hovering honeybee, Apis mellifera [16]: positional angle (stroke angle), elevation angle (deviation angle) and featuring angle (geometric angle of attack) are plotted as in Fig 4 (b). The body angle $\chi$ is 45.0 deg., the stroke plane angle $\beta$ is $0 \mathrm{deg}$, the wing stroke amplitude $\Phi$ is $90.5 \mathrm{deg}$., and the flapping frequency $f$ is $229.8 \mathrm{~Hz}$. Therefore, the Reynolds number $(R e)$ is estimated to be approximately 1,123 and the reduced frequency $(k)$ is 0.244 . It should be mentioned that the wing stroke amplitude is observed in most insects over a range of 145-160 deg., a bee, however, usually hovers with relatively shallow strokes less than $130 \mathrm{deg}$. separated by rapid reversals [16].

\section{Fruitfly hovering: $R e=100-500$}

A realistic wing-body model of a fruitfly, Drosophila melanogaster [9], is built: a body length is $2.78 \mathrm{~mm}$, a wing length $(R)$ is $2.39 \mathrm{~mm}$ and a mean chord length $\left(c_{m}\right)$ is 0.78 mm. A $0.008 c_{m}$ flat plate wing model is employed with a virtual portion of $0.03 c_{m}$ added to the wing base. Grid system of the wing-body fruit fly model as illustrated in Fig 2 (b-3) is composed of three-blocked overset grids (right wing grid: 62,775 points, left wing grid: 62,775 points, body grid: 200,925 points). O-O type structured body-fitted grid is employed. Kinematic models are based on the experimental data of a hovering fruitfly, Drosophila melanogaster [7] [14] : positional, feathering and elevation angles as in Fig 4 (c); the body angle $\chi$ is $45.0 \mathrm{deg}$; the stroke plane angle $\beta$ is $0 \mathrm{deg}$; wing stroke amplitude $\Phi$ is 139.8 deg.; and the flapping frequency $f$ is $218.0 \mathrm{~Hz}$. Thus, the Reynolds number $(R e)$ and the reduced frequency $(k)$ are calculated to be 134 and 0.212 , respectively.

\section{Thrips hovering: $R e=5-20$}

A wing-body model of a thrips is based on the image data of a thrips, franklinella intonsa $[9,18]$ : a body length is approximately $0.8 \mathrm{~mm}$, a wing length $(R)$ is $0.5 \mathrm{~mm}$ and a mean chord length $\left(c_{m}\right)$ is $0.384 \mathrm{~mm}$ [18]. It should be noted that the four-winged 
thrips may be modeled with a body and a wing pair (Fig 2 (b-4)) while any wing-wing interference effects are neglected under this assumption. This is thought reasonable with consideration of the fact that movement of the fore-and hind-wing is nearly analogous [3]. Due to the complexity and difficulties in morphological modeling in terms of meshing the bristled wing is modeled as a flat plate with uniform-thickness of $0.012 c_{m}$. The experimental results reported by Sunada et al. [18] have pointed out that at ultra low Reynolds numbers on the order of 10 a bristled wing showed distinguished aerodynamic characteristics from those performed by a membrane wing. Although the present approximation in wing-geometry modeling may not be appropriate, it could provide a quantitative prediction on how a flapping membrane wing works on aerodynamic forcegeneration at very low Reynolds number and furthermore, on how different this may be compared with a bristled flapping wing. Kinematic models of a hovering thrips are built based on the experimental data of a hovering fruitfly, Drophila ([19]) because there are no available experimental data regarding the flapping wing and body kinematics and the wing kinematics of thrips are observed to be similar to flapping small insects such as a fruitfly. The fruitfly-like hovering wing kinematics is illustrated in Fig 4 (d): positional, feathering, and elevation angles; the body angle $\chi$ is $90.0 \mathrm{deg}$; the stroke plane angle $\beta$ is 0 deg; wing stroke amplitude $\Phi$ is 139.8 deg.; and $f=200.0 \mathrm{~Hz}$. Accordingly, the Reynolds number $(R e)$ is calculated to be 12 and the reduced frequency $k$ is 0.2485 .

Non-dimensional parameters including the stroke plane and body angle, the aspect ratio, the Reynolds number and the reduced frequency for typical four insects are summarized in Table 1.

\section{Results and Discussion}

Although wings of biological flyers are flexible structure, the study based on the rigid wing framework shares fundamental basis of flapping wing aerodynamics and can offer useful insights. In particular, the Reynolds number effect associated with the change of size of flyers on the aerodynamics is one of interests to be important for understanding flapping wing aerodynamics [9]. Therefore, we will highlight the size or Re effects on flapping wing aerodynamics based on the results obtained from rigid-wing framework. 
Note that recently many efforts are made using experimental or numerical flexible wing framework (e.g. [1, 21, 22, 23, 24]).

\subsection{Size effects on vortex dynamics and wake structures}

Comparison of instantaneous coherent vortical structures around four typical insects in hovering flight present pronounced discrepancy due to the effect of size or $R e$ in terms of vortex dynamics and vortical structures (Fig 5). The flow structures around the flyers operating at Re of $O\left(10^{1}\right)$ and $O\left(10^{2}\right)$, e.g., the fruitfly and the thrips, due to increase of viscous damping the horse-shoe vortices (HSV) consisting of the LEV, the TEV, and the TV present less three-dimensionality and planar smooth vortex rings during the downand up-stroke. The LEV attaches on the wing without breaking down throughout the one stroke. On the other hand, in the high Reynolds number cases of hawkmoth and honeybee hovering, the LEV, however, concentrates its vorticity into an intense, conically-shaped one and breaks down at approximately $70-80 \%$ wing length from the wing root near the mid-late downstroke, resulting in an enlarged complicated vortex system observed near the wing tip [10]. This discrepancy in overall vortical structures due to Re effect are observed in a recent experimental flow measurement [25] that investigated Re effect on vortical structures around a rotating plate with constant angle of attack (45 deg.) based on particle image velocimetry (PIV). Their results presented that the vortex structure of $R e$ of 60 are quite different form that of $R e$ of 8,800 due to vorticity distribution by diffusion, the LEV and TV are more widely distributed for $R e$ of 60 , and the vortex structure of $R e$ of 1,100 is similar to that of $R e$ of 8,800 .

Nevertheless, the computed and experimental results confirm that the LEV phenomenon is universal in flapping flight, which can be seen at all sizes or the Reynolds numbers. However, the LEV does show different development and structure at different sizes or Reynolds numbers, which largely depends upon sizing other than morphology and/or wing kinematics. As illustrated in Fig 5, the LEV and the vortex ring are quite similar in shape and strength in the cases of hawkmoth and honeybee hovering though these exists a distinguished discrepancy in the wing kinematics. Hovering with the low stroke amplitude and the high flapping frequency the honeybee ([16]) has a mean wingtip speed $(3.6 \mathrm{~m} / \mathrm{s})$ is 1.5 times greater than that of the hawkmoth $(2.6 \mathrm{~m} / \mathrm{s})$. This indicates that, since both wingtip speed and stroke amplitude affect the transient LEV formation 
the relatively low stroke amplitude (i.e. a shorter distance of wing translation) may be able to create a strong and stable LEV to help generating sufficient force for the honeybee hovering ([16]). An experimental study by Altshuler et al. [16] on effects of the stroke amplitude on the force-generation with a scaled robotic-wing presented that increasing the stroke amplitude while maintaining constant frequency (i.e. at the same mean wingtip speed) could increase the contribution of aerodynamic forces generated during the translation motion of the wing. On the other hand, the mean wingtip speed in the fruitfly hovering is calculated to be $1.3 \mathrm{~m} / \mathrm{s}$, lower than those of the hawkmoth and the honeybee, and the LEV keeps stable without breaking-down throughout the half stroke. Recent three-dimensional flow measurement with dye visualization [26] and phase-locked and phase-averaged stereoscopic PIV [27] provided further understanding of transient LEV formation generated by a rotating flat plate with fixed angle of attack (45 deg.) in terms of the LEV and TV interaction at Re of 5,000. Their results revealed that the influence of the TV is relatively large for low $A R$ in comparison with the $A R$ of 4 and the TV anchors the LEV in the tip region [26, 27, 28]. Furthermore, numerical simulations of the $A R$ of 4 flat plate for Re of 1,000 [32] and 100 [29] also observed similar $\mathrm{TV}$ and LEV interaction around the flapping wing undergoing rectilinear translation.

While the LEV significantly contributes to the force-generation on flapping wings independent on sizing or Reynolds number, the mechanism of its stability is still under discussions. It may be fair to suggest that the pressure-gradient force, the centrifugal force, and the Coriolis force together play a role in but work differently in enhancing the LEV stability in terms of sizing effect. In present study we focus on the pressure-gradient effect and illustrate pressure-gradient contours on the flapping wings of a fruitfly, a honeybee, and a hawkmoth (Fig 6). The computed pressure-gradient contours obviously show greater magnitude at high Reynolds numbers (i.e. the hawkmoth and the honeybee) while smaller at low Reynolds number (i.e. the fruitfly). This suggests that the pressuregradient may work differently at different Reynolds numbers on causing the axial flow at the LEV core [7] [10] [17] and on enhancing the stability of the LEV. At low Reynolds numbers of $O\left(10^{2}\right)$ or less, e.g., for a fruitfly, the flapping wing may not be able to create an LEV strong enough to generate a steep pressure-gradient at the LEV core. Recently, Ozen and Rockwell [31] studied the flow structure around a rotating $A R$ of 
1 flat plate with constant angular velocity after $270 \mathrm{deg}$. of rotation from the startup, well after transient effect subside. They investigated $R e$ and angle of attack ranges of 3,600-14,500 and 30 deg. to 75 deg., respectively and found a stable LEV for all cases and little variation with Re. They observed that the size of the LEV and normalized circulation of LEV increase with increasing the angle of attack. Moreover, they showed that the stable LEV is linked to significant velocity directed toward the leeward surface of the plate, between the LEV and trailing edge, in addition to substantial spanwise flow and velocity toward the plate coming from the root to TVs. Furthermore, they also found fully separated flow without the attached LEV around a translating plate with equivalent motion. Moreover, Jardian, Farcy, and David [32] compared the flow that developed around a revolving and translating wing for Re of 1,000. They found that the position of the connecting points between the LEV and the TV matches the limit of the three-dimensional region observed in the translating case. The resign of strong inward velocities induced by the TV (in translating case) corresponds to that of strong outward velocities induced by the spanwise incident velocity gradient (in the revolving case). Therefore, inward velocities counteract outward velocities, giving rise a local instability. As such the LEV detaches near the wing tip but can keep attached along $85 \%$ of the wing span. This promotes the tilting of the LEV into the stream wise direction. The LEV and TV being co-rotating, the connect into single entity. Beyond the connecting point (towards the wing tip), the flow is unstable and significantly affected by wing-wake interactions. Before the connecting point, the flow is stable and weakly affected by wing-wake interactions. In this stable region, several studies [4, 33] suggested that spanwise flow balances vorticity production at the leading edge, hence allowing the continuous attachment of the LEV.

The downwash wake structures also illustrate pronounced sizing or Reynolds number dependency. In Fig 7, front views present vertically pulsed lattice downwash wakes, synonymous with the shedding and diffusion of the vortex ring wake at downstroke; top views further reveal that the cores of the momentum jets (or, the jet columns) oscillate horizontally. The hawkmoth hovering shows two largely distorted momentum jet columns, which, in fact, link together, resulting in a pronounced wider downwash disc and hence a relatively narrower low-speed zone right below the body. The jet 
stream columns are observed to depart when the size or Reynolds number goes down, e.g., for honeybee and fruitfly, leaving a low-speed or dead-fluid zone beneath the body. Furthermore, recent experimental study revealed that downwash wake structures around hovering hummingbirds (similar Re of the hawkmoth) is similar to that of the hawkmoth hovering [34] and that of small birds becomes a single down momentum jet because one vortex ring generated by two-wings per stroke [35].

\subsection{Size effects on aerodynamics performance}

Time courses of vertical forces over one flapping cycle in hovering flight of the four insects are plotted in Fig 8. In Fig 8 the forces on the two wings (red) and the body (blue) are shown. Pronounced discrepancy in vertical force generation can be observed that, in hawkmoth hovering two peaks are seen in each stroke however in frutifly and thrips hovering a single peak is seen while in a honeybee hovering double peaks in the downstroke and a single peak in the upstroke is observed. Since the LEV is observed throughout a complete stroke cycle in all the four cases (Fig 5), the LEV is very likely responsible for most of the force generation at all sizes or the Reynolds numbers. The downstroke-and upstroke vortex ring initiated from a HSV is observed for three insects except the tiny thrips, which is compact and stable throughout the half stroke. It is not clear whether this stable vortex ring can stabilize the LEV and hence enhance the fore-generation, but it is reasonable to hypothesis that this vortex ring may be responsible for stabilizing the TV and even the TEV, which is also important for the LEV because they are linked together. In a sense, the stable vortex ring itself is likely a key in augmenting the forcegeneration of every half stroke. So how this vortex ring links to the force-generation at different Reynolds numbers? The two peaks at high Reynolds number (hawkmoth and honeybee) or the one peak at low Reynolds numbers (fruitfly and thrips) may be an integrated result of the break down at late-half stroke and/or added mass effect due to flapping wing kinematics, which, including which peaks correspond with which vortex dynamic events, needs to be further confirmed in the future. In hawkmoth hovering (Fig 5 and Fig 8), we do find that at early downstroke when the LEV is still not strong sufficient the starting vortex, i.e. the shed TEV connecting to the TV keeps its strength and results in a low pressure at the wing tip which corresponds to the first force peak (Fig 8). This implies that not only the LEV but also the TEV and TV can contribute 
to the force-generation in flapping-wing flight, which may depend upon wing kinematics and size [7]. In honeybee hovering, however, the time course shows prominent difference where two additional but smaller force peaks are observed during beginning and end of each stroke, which as suggested by Altshuler et al. [16] may indicate the importance of additional unsteady mechanism at stroke reversal. This, however, seems to be Reynolds number-dependent. At low Reynolds numbers, in fruitfly hovering (Fig 5 and Fig 8) and, in particular, thrips hovering (Fig 5 and Fig 8), the TEV and TV become much more weaker and this mechanism never works anymore, but instead a rapid rotation of the flapping wing may result in a rapid increase in circulation, possibly also leading to augmenting the force production.

A ratio of cycle-averaged vertical force generated in each stroke is further example of the effect of size or Reynolds number: approximately 6:4-7:3 in hawkmoth hovering, 5:5 in honeybee hovering, 4:6 in fruitfly hovering, respectively. For birds, Warrik et al. [36] have reported that this ratio is 7.5:2.5 in the hovering flight of hummingbird. This obviously indicates a strong size or Reynolds number dependency in the aerodynamic force generation, which implies that the flapping wing kinematics should play a key role. Interestingly, in comparison with fruitfly and honeybee hovering, while they have almost the same stroke plane angle and the body inclination angle, their time courses of the vertical forces are obviously different, which, as discussed previously, may be due to the fact that honeybees hover with low stroke amplitude and high wing-beat frequency as well as flying in higher Reynolds number condition.

The computed mean vertical force generated by the thrips model $\left(1.03 \times 10^{-8} \mathrm{~N}\right)$ is only $17.2 \%$ of its weight (approximately $6.0 \times 10^{-7} \mathrm{~N}[18]$ ). Although qualitative comparison between time histories of computation [9] and experiment [20] has been in reasonable agreement, this discrepancy of mean and time-varying lift force among the computation, the robotic experiment, and real thrip of hovering flight provides interesting topics for analyzing flapping wing aerodynamics under very low Reynolds number conditions. First, as seen in Fig 5, flow structures around the tiny thrips show less three-dimensionality and the aerodynamics at ultra low Res ranging over 1 to 100 do present somehow drastic difference, even a big jump in-between a range when the Reynolds number drops from 50 down to 10 or lower $[37,38]$. In a sense, aerodynamics may behave quite differently 
at a Reynolds number around 10 or lower. The viscous force obviously dominates such low Reynolds number flows: the LEV and the TEV is difficult to develop and affect a significant fraction of the wing area (Fig 5); the flow around the plate is severely slowed which results in a substantially large skin friction. Secondary, development of the realistic wing configuration and kinematics models are required for both computations and experiments. As already mentioned the wing configurations and wing kinematics of the current computation are different from those of real thrips. Current study models the bristled wing structure as a rigid flat plate and employes the flapping wing kinematics of a fruitfly but not a thrips. Sunada et al. [18] reported that measured fluid-dynamic forces acting on a bristled model wing were a little smaller than those of the flat plate model wing at ultra low Reynolds numbers. However, force generation mechanisms associated with the bristled wing are still not very clear yet. Furthermore, the present rigid and flat plate wing model is based on the image of thrips $[9,18]$, in which an effective wing shape and hence the area including the hairs attached on the wing is very likely not constructed reasonably. The front and hind wings usually flaps with some phase difference, which can result in a cambered wing and may enhance the aerodynamic performance. Since the thrips is tiny insect kinematics of hovering thrips including front-and hind-wing and body movement, and phase difference or chamber is still not available due to the difficulty of measurement. If any exist, may be further a key issue in dominating the aerodynamic force-generation in the flapping bristled wings of thrips. The stroke plane angle and the body inclination angle may also impact on the aerodynamic force-generation. Thirdly, the incompressible assumption may be invalid and moreover the no-slip condition may also fail in ultra low Reynolds number flows. Sun and Boyd [38] studied aerodynamics at very-low Reynolds numbers between 1 and 200 by simulating gas flows around a $5 \%$ flat plate using a hybrid continuum-particle approach. They concluded that the low Re flows are viscous and compressible, and rarefied effects (a velocity slip exists on the solid surface for flows in the slip regime where the Knudsen number (the ratio of the mean free path of gas molecules to the smallest characteristic length of the flow) is between 0.01 and 0.1) increase when the Reynolds number decreases. Given the mean chord length $\left(c_{m}=0.384 \mathrm{~mm}\right)$ of thrips as characteristic length a global Knudsen number is calculated to be 0.00015; while a local Knudsen number based on the diameter (e.g. 10 micron) 
of single hair of a thrip wing is approximately 0.005 . Hence, the rarefied effects may be important for ultra low Reynolds number aerodynamics. This, if is the case, would definitely enhance the difficulties in the modeling, which would make the computation much more expensive because continuum equations (Navier-Stokes equations) could not describe the flow and the kinetic-based approaches, or, some hybrid-methods (e.g. [39]) may need to be introduced. Nevertheless, the flapping wing aerodynamics at such ultra low Reynolds numbers leaves us a fascinating topic: why thrips have bristled but not membrane wings as they do, which very likely leads to discovery of some novel aerodynamic mechanisms distinguished from those at moderate Reynolds numbers. All these considerable factors needed to be addressed to reveal aerodynamic mechanisms under very low Reynolds number conditions.

\section{Conclusions}

Current study analyzed sizing or Re effects on insect hovering aerodynamics and offered an overview of scaling effects on vortex dynamics, wake structures and their relationship with force-generation over a wide range of Res from $\mathrm{O}\left(10^{1}\right)$ to $\mathrm{O}\left(10^{4}\right)$. The results revealed how the LEV is related to the TEV and the TV as well as to a vortex ring and pointed to the importance of the vortex ring in stabilizing the LEV and hence in enhancing the force-generation. Furthermore, some open challenges and important points for analyzing flapping wing aerodynamic under very low Reynolds number conditions were highlighted.

\section{Acknowledgment}

This work is partly supported by a PRESTO-JST program, the Grant-in-Aid for Scientific Research of No. 18656056 and No. 18100002, JSPS, Japan.

\section{References}

[1] W. Shyy, L. Yongsheng, J. Tang, D. Viieru, H. Liu, Aerodynamics of Low Reynolds Number Flyers Cambridge Aerospace Series (2008).

[2] J.M. McMasters, M.J. Henderson, Technical Soaring 61 (1980).

[3] T. Weis-Fogh, Journal of Experimental Biology 59:169-230 (1973). 
[4] C.P. Ellington, C. van den Berg, A.P. Willmott, A.L.R. Thomas, Nature 384: 626-630 (1996).

[5] M.H. Dickinson, F.-O. Lehmann, S.P. Sane Science 284: 1954-1960 (1999).

[6] F.M. Bos, D. Lentink, B.W. Van Oudheusden, H. Bijl, Journal of Fluid Mechanics 594:341-368 (2008).

[7] H. Aono, F. Liang, H. Liu, Journal of Experimental Biology 211:239-257 (2008).

[8] W. Shyy, H. Aono, S.K. Chimakurthi, P. Trizila, C.-K. Kang, C.E.S. Cesnik, H. Liu, Progress in Aerospace Sciences 46: 284-327 (2010).

[9] H. Liu, H. Aono, Bioinspration and Biomimetics 4: 015002 (2009).

[10] H. Liu, C.P. Ellington, C. van den Berg, K. Kawachi, A.P. Willmott, Journal of Experimental Biology 201: 461-477 (1998).

[11] H. Liu, K. Kawachi, Journal of Computational Physics 146: 124-156 (1998).

[12] H. Liu, Journal of Computational Physics 228: 439-459 (2009).

[13] A.P. Willmott, C.P. Ellington, Journal of Experimental Biology 200: 2705-2722 (1997).

[14] S.N. Fry, R.Sayaman, M.H. Dickinson, Journal of Experimental Biology 208: 2303-2318 (2005).

[15] L. Alford, A. Altman, SAE Aerospace Division Conference, Reno, NV, Society of Automotive Engineers Paper 2004-01-3089 (2004).

[16] D.L. Altshuler, W.B. Dickson, J.T. Vance, S.P. Roberts, M.H. Dickinson Proceedings of the National Academy of Sciences of the United States of America 102: 18213 (2005).

[17] J.M. Birch, W.B. Dickson, M.H. Dickinson, Journal of Experimental Biology 207: 1063-1072 (2004).

[18] S. Sunada, H. Takashima, T. Hattori, K. Yasuda, K. Kawachi, Journal of Experimental Biology 205: 2737-2744 (2002)

[19] J.M. Zanker, Philosophical Transactions of the Royal Society B 327: 1-18 (1990).

[20] F.-O. Lehmann, S.P. Sane, M.H. Dickinson, Journal of Experimental Biology 208: 3075-3092 (2005).

[21] L.A. Miller, C.S. Peskin, Journal of Experimental Biology 208:195-212 (2005).

[22] J. Young, S.M. Walker, R.J. Bomphrey, G.K. Taylor, A.L.R. Thomas, Science 325: 1549-1552 (2009).

[23] C.-K. Kang, H. Aono, C.E.S. Cesnik, W. Shyy, Journal of Fluid Mechanics 689: $32-74$ (2011).

[24] T. Nakata, H. Liu, Philosophical Transactions of the Royal Society B 279: 722-731 (2011)

[25] D. Kim, M, Gharib, Experiments in Fluids 49: 329-339 (2010).

[26] A.C. Devoria, P. Mahajan, M.J. Ringuette, AIAA paper 2011-396 (2011).

[27] Z. Carr, C. Chen, M.J. Ringuette, AIAA paper 2012-912 (2012).

[28] M.J. Ringuette, M. Milano, M. Gharib, Journal of Fluid Mechanics 581:453-468 (2007).

[29] W. Shyy, P. Trizila, C.-K. Kang, H. Aono, AIAA Journal 47: 289-293 (2009).

[30] J.M. Birch, M.H. Dickinson, Nature 412:729-733 (2001).

[31] C.A. Ozen, D. Rockwell, Experiments in Fluids 52:207-223 (2012).

[32] T. Jardin, A. Farcy, L. David, Journal of Fluid Mechanics 702: 102-125 (2012).

[33] D. Lentink, M.H. Dickinson, Journal of Experimental Biology 212: 2705-2719 (2009).

[34] D.L. Altshuler, M. Princevac, H. Pan, J. Lazano, Experiments in Fluids 46: 835-846 (2009).

[35] Y.-H. Chagn, S.-C. Ting, C.-C. Liu, J.-T. Yang, C.-Y. Soong, Experiments in Fluids 51:1231-1243 
(2011).

[36] D.R. Warrick, B.W. Tobalske, D.R. Powers, Nature 435: 1094-197 (2005).

[37] L.A. Miller, C.S. Peskin, Journal of Experimental Biology 207:3073-3088 (2004).

[38] Q. Sun, I. D. Boyd, Journal of Fluid Mechanics 502: 199-206 (2004).

[39] Q. Sun, I. D. Boyd, G.V. Candler, Journal of Computational Physics 194: 256-277 (2004). 


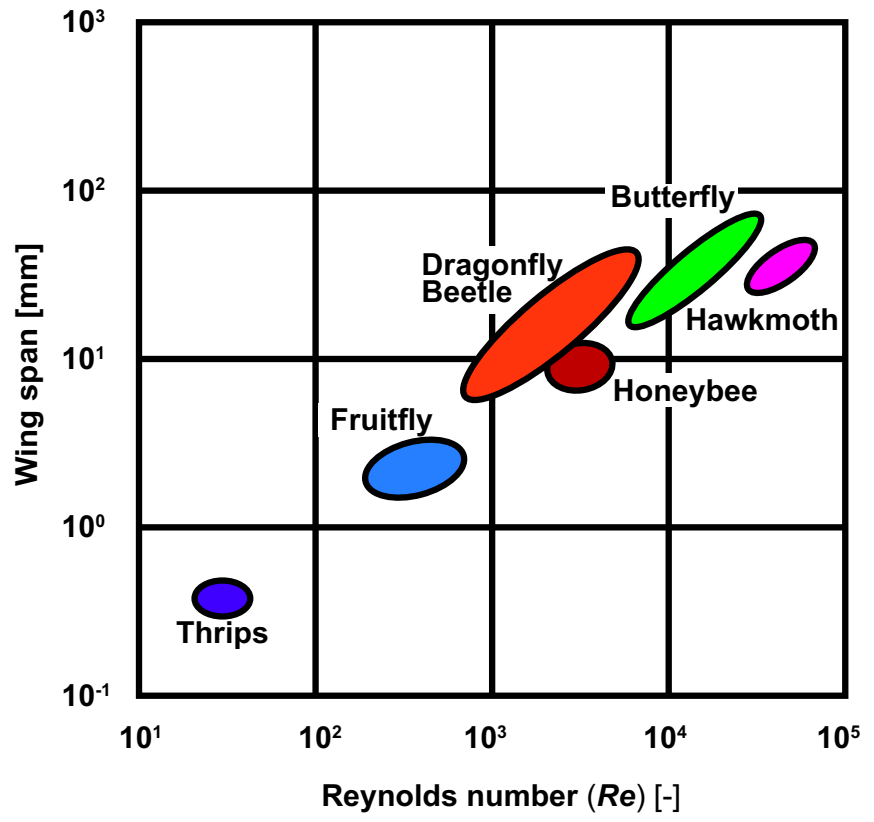

Figure 1: Diagram of relationship between Reynolds number and wing span in biological flights. 
(a)

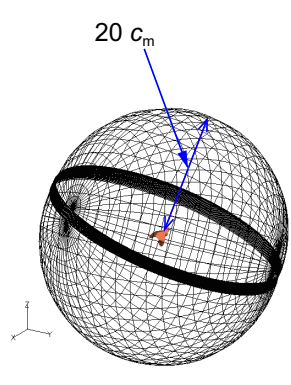

(b-1)

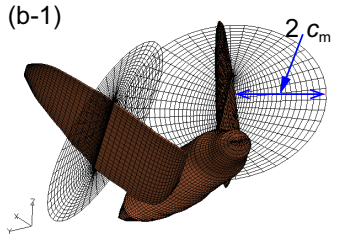

(b-3)

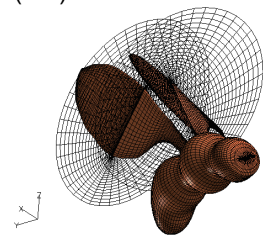

(b-2)

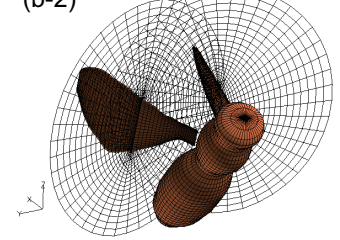

(b-4)

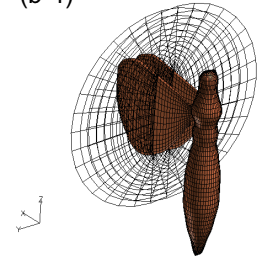

Figure 2: Computational grid system of morphological models: (a) overview, (b-1) a hawkmoth, (b-2) a honeybee, (b-3) a fruitfly, and (b-4) a thrips. 


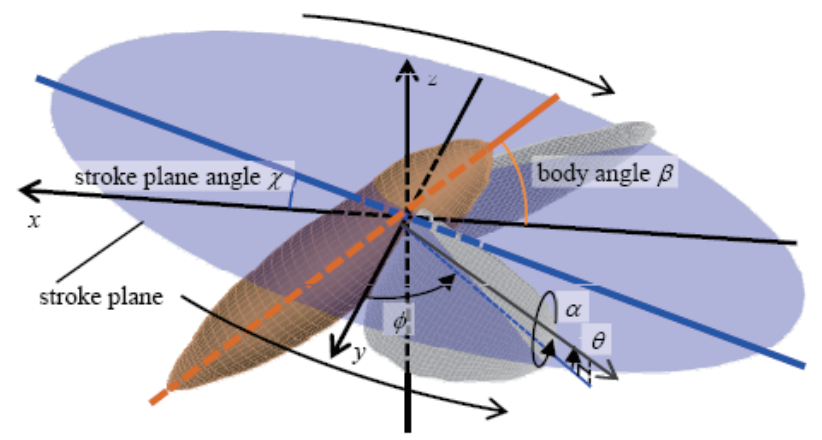

Figure 3: Schematics of basic rotational angles of flapping wing motions, stroke plane angle, and body angle. 

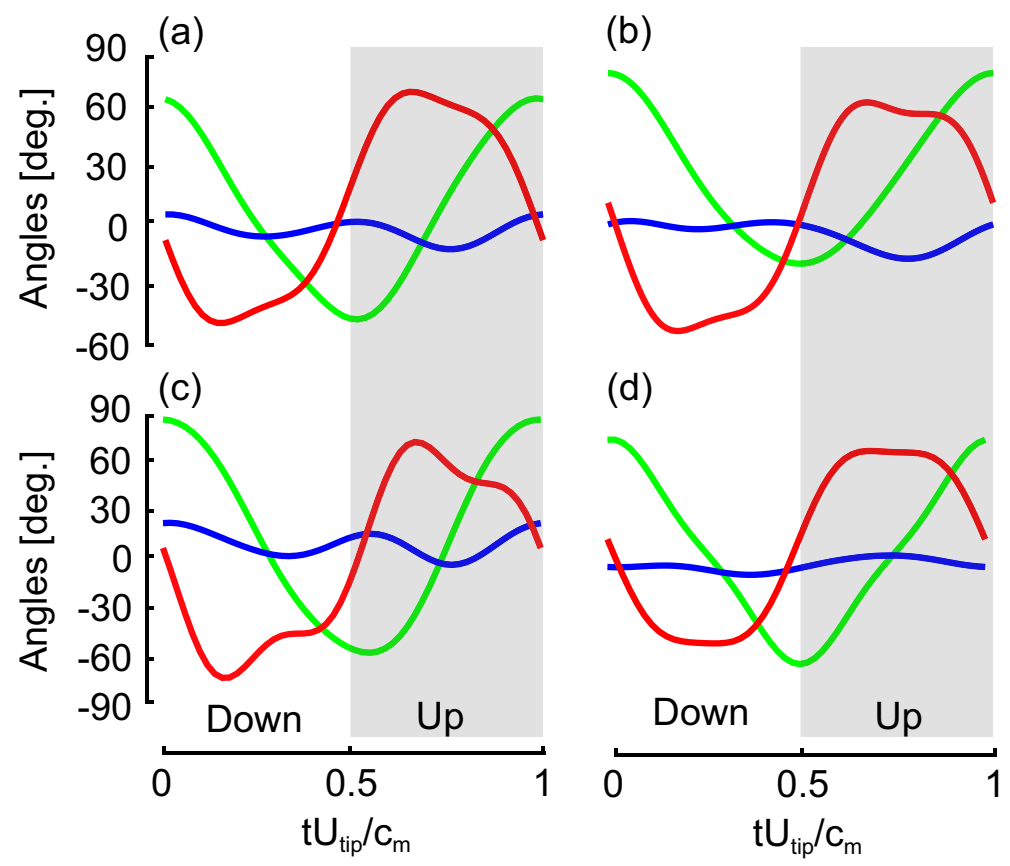

Figure 4: Time histories of wing kinematic models in hovering flight: (a) a hawkmoth, (b) a honeybee, (c) a fruitfly, and (d) a thrips. Red, blue, and green solid lines correspond to the positional/stroke angle $(\varphi(t))$, the elevation/deviation angle $(\vartheta(t))$, and the feathering angle $(\alpha(t))$ 


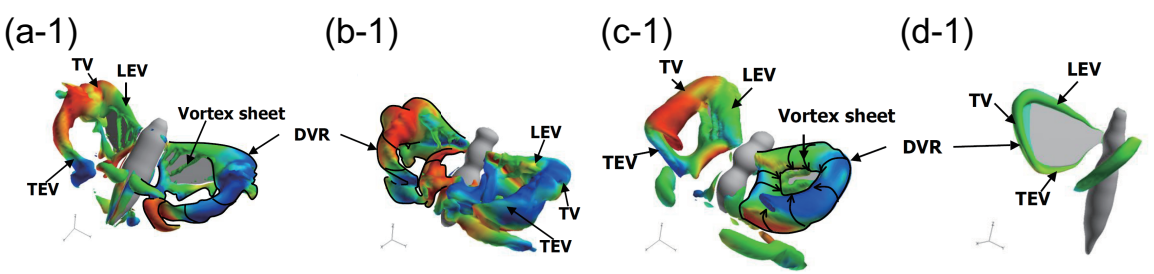

Downstroke

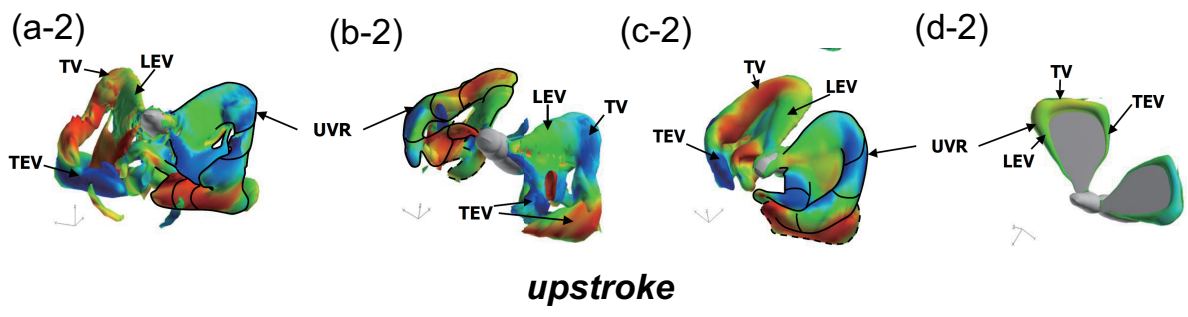
(a) Hawkmoth
(b) Honeybee
(c) Fruitfly
(d) Thrips

Figure 5: Instantaneous vortex structures around (a) a hovering hawkmoth, (b) a hovering honeybee, (c) a hovering fruitfly, and (d) a hovering thrips at the middle of the down-and up-stroke. Iso-surface indicates the vorticity magnitude and is colored by normalized helicity density. LEV, TEV, TV, DVR, and UVR indicate leading edge vortex, trailing edge vortex, tip vortex, downstroke vortex ring, and upstroke vortex ring, respectively. 
(a) Hawkmoth

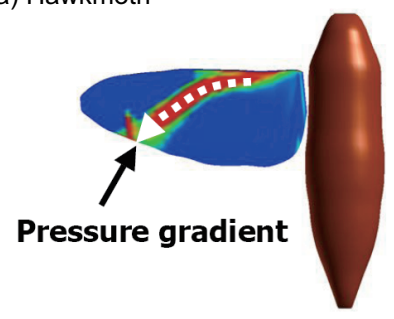

(c) Fruitfly

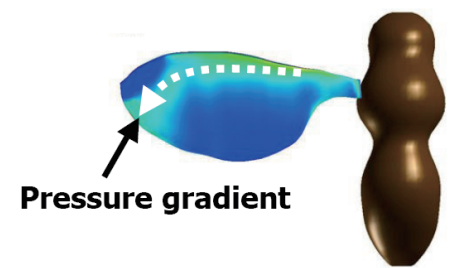

(b) Honeybee

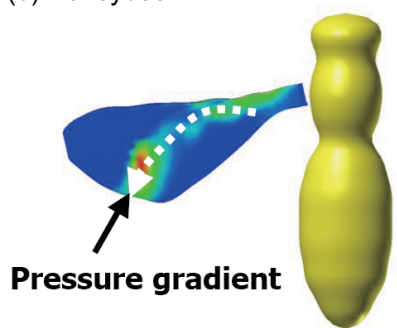

\section{Normalized pressure gradient

$\begin{array}{lll}0 & 1 & 2\end{array}$ \\ Normalized by $c_{m} /\left(\rho_{\text {air }} U_{\text {tip }}\right)^{2}$}

Figure 6: Distribution of spanwise pressure gradient of (a) a hovering hawkmoth, (b) a hovering honeybee, and (c) a hovering fruitfly. 


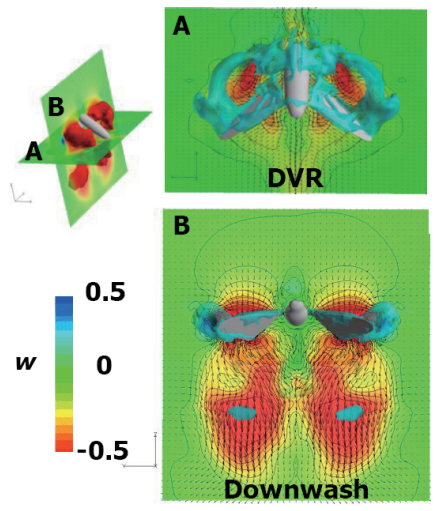

(a) Hawkmoth

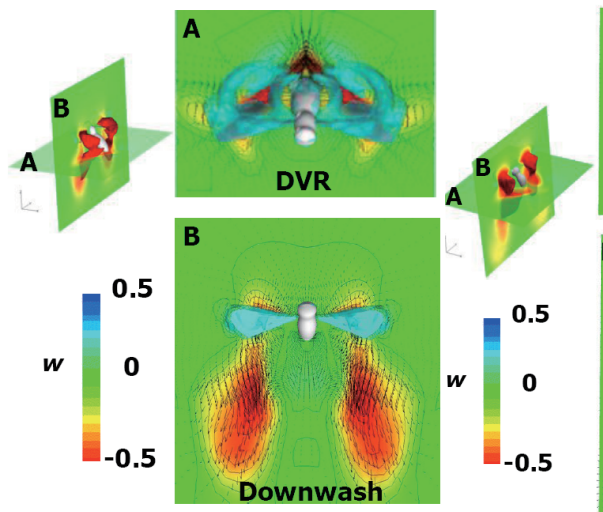

(b) Honeybee

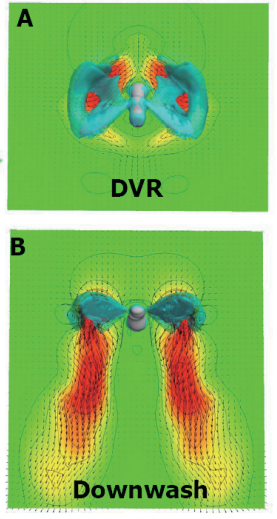

(c) Fruitfly

Figure 7: Instantaneous snapshots of wake structures generated by (a) a hovering hawkmoth, (b) a hovering honeybee, (c) a hovering fruitfly near the middle of downstroke. 

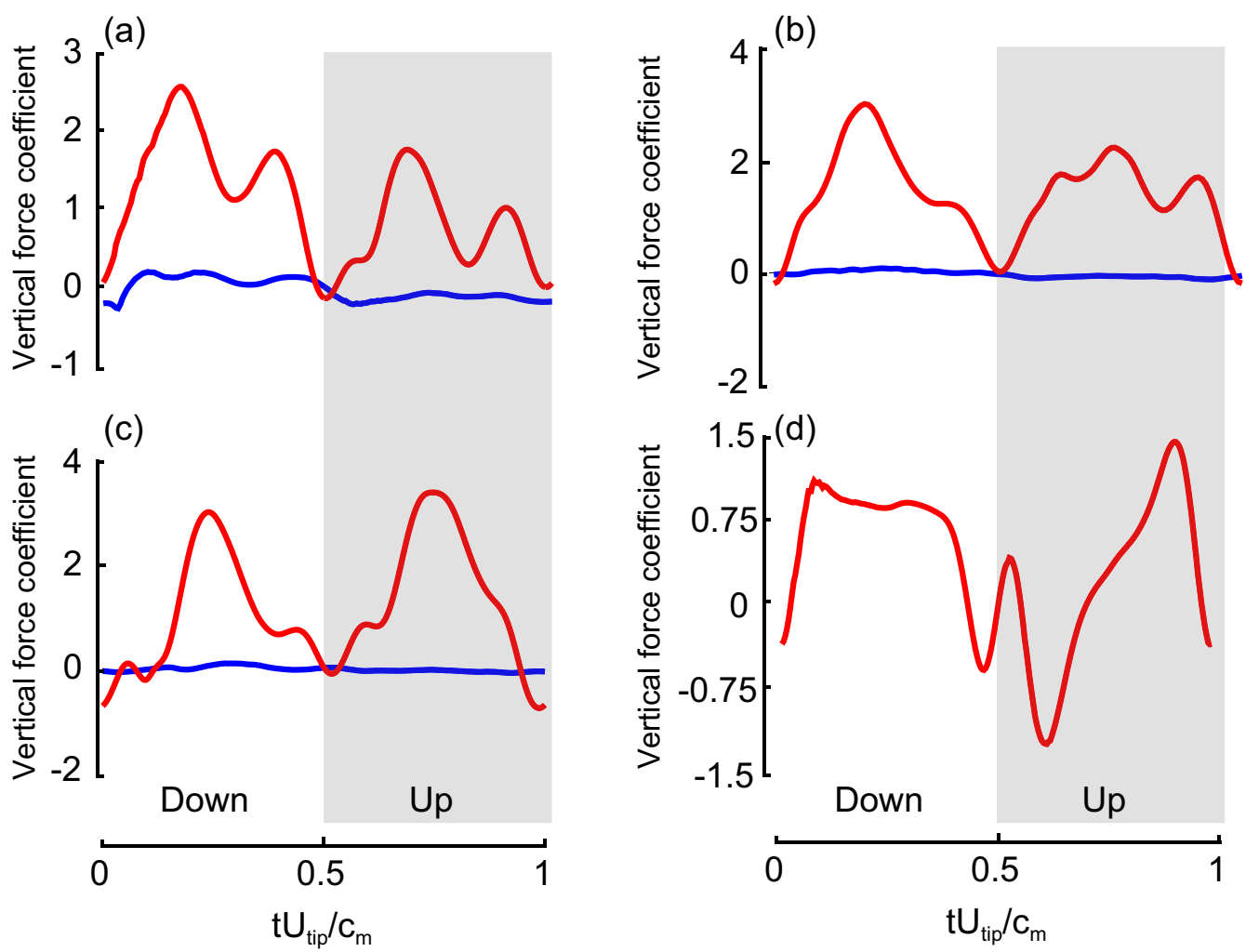

Figure 8: Time histories of vertical force over one flapping cycle in hovering flight : (a) a hawkmoth, (b) a honeybee, (c) a fruitfly, and (d) a thrips. Red and blue solid lines indicate vertical force acting on two wings and body. 


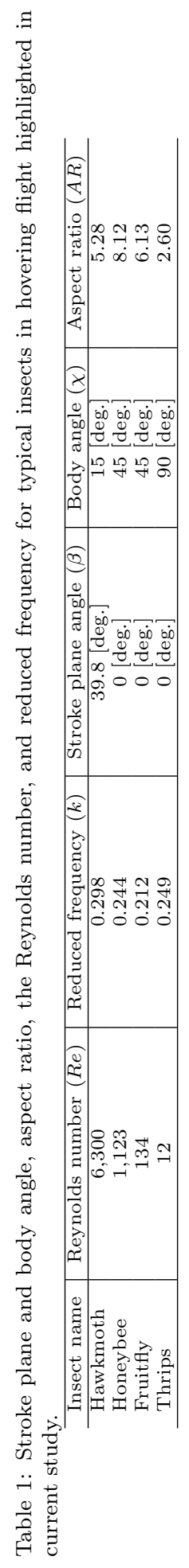

